\title{
The mesothelioma surgery shift
}

\author{
Raja M. Flores, MD
}

\footnotetext{
From the Department of Thoracic Surgery, Icahn School of Medicine, Mount Sinai Health System, New York, NY. Disclosures: Author has nothing to disclose with regard to commercial support.

Received for publication Oct 19, 2015; accepted for publication Oct 19, 2015; available ahead of print Nov 24, 2015.

Address for reprints: Raja M. Flores, MD, Department of Thoracic Surgery, Mount Sinai Health System, Icahn School of Medicine at Mount Sinai, One Gustave L. Levy Place, Box 1023, New York, NY 10029 (E-mail: raja.flores@mountsinai.org).

J Thorac Cardiovasc Surg 2016;151:485-6

$0022-5223 / \$ 36.00$

Copyright (C) 2016 by The American Association for Thoracic Surgery

http://dx.doi.org/10.1016/j.jtcvs.2015.10.054
}

There has been a general shift in surgery for mesothelioma from extrapleural pneumonectomy (EPP) to pleurectomy and decortication (PD) after a large comparative multicenter study by experienced mesothelioma surgeons failed to demonstrate great survival differences between the 2 procedures. ${ }^{1}$ Even the Mesothelioma and Radical Surgery (MARS) group has shifted its focus from EPP to PD by embarking on another randomized, controlled trial comparing PD with nonsurgical treatment. The shift from EPP to PD requires more than just a simple shift in operative technique, it requires a prodigious shift in mindset and a refocusing on the goals of surgery.

Macroscopic complete resection (MCR) is a term coined by the EPP pioneer David Sugarbaker, ${ }^{2}$ who stated that this was to be the primary goal of surgical resection in mesothelioma. At first, this term appears to deviate from the standard oncologic terminology of R0, R1, and R2, but MCR is essentially just an R1 resection. In the case of most solid tumors, R1 is a suboptimal result. So why should this be the sole primary goal of surgery? Is this determination based on sound survival data?

The study from the Turkish group Batirel and colleagues ${ }^{3}$ in this issue of the Journal provides a table of relevant studies evaluating survival with surgical resection. The MCR rationale was championed primarily on the basis of the Sugarbaker study cited in this table. ${ }^{3}$ This study encompassed a highly selected group of only patients with epithelioid disease who underwent only EPP with no comparison group. Patients with incomplete resections, those with PD, and those with nonepithelioid mesothelioma were excluded from the analysis. Median survival was 18 months. When one analyzes the other studies on the same table, ${ }^{3}$ one quickly recognizes that the MCR (R1) rationale for EPP does not translate into the best overall survival.

Observing 2 paradoxes in the Turkish study further highlights this struggle regarding the association of survival with optimal cytoreduction. (1) The shift from EPP to PD was associated with a decrease in $\mathrm{R} 1$ (MCR) resection from $65 \%$ to $41 \%$. (2) In the EPP group, $87 \%$ of the

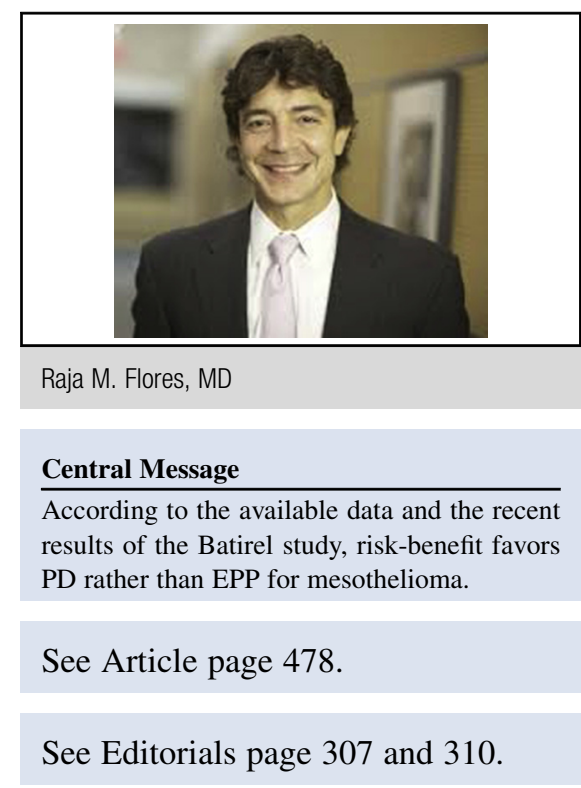

patients had epithelioid histologic type, whereas only $59 \%$ in the PD group had epithelioid histologic type. These 2 findings should translate into a survival difference in favor of EPP, but no such difference was observed. The nihilist from afar might interpret this to mean that both procedures are ineffective, or the surgeon in the trenches might interpret these findings to mean that the adverse effects of EPP offset the benefits of the cytoreduction it provides. The downside of EPP may not lie just in a higher operative mortality but also in the general higher mortality during the first postoperative year and beyond of patients who have undergone pneumonectomy, and in the quicker time to death of patients with recurrent mesothelioma after EPP than after PD. ${ }^{1,4}$

The data suggest that the primary goal of surgery should not just be to obtain an MCR (R1) at the expense of pneumonectomy. More realistic goals should include the removal of as much tumor as possible while avoiding pneumonectomy, lung reexpansion, prevention of fluid reaccumulation, while minimizing morbidity and mortality.

The mesothelioma risk-benefit equation may look something like this: Gain in survival time = (time gained by cytoreduction + time gained by lung reexpansion) (time lost by operative mortality - time lost by long-term mortality of surgical procedure - time lost from accelerated death after recurrence).

On the basis of the currently available data, including the most recent results of the study of Batirel and colleagues, ${ }^{3}$ the equation tips in favor of PD rather than EPP. A randomized, controlled trial is not necessary to answer 
this question. The take-home message is analogous to the concept of the law of diminishing returns; remove as much tumor as possible but without sacrificing the lung. New drug discoveries may bring us full circle again in the future. But we have our answer-for now.

\section{References}

1. Flores RM, Pass HI, Seshan VE, Dycoco J, Zakowski M, Carbone M, et al Extrapleural pneumonectomy versus pleurectomy/decortication in the surgical management of malignant pleural mesothelioma: results in 663 patients. J Thorac Cardiovasc Surg. 2008;135:620-6.

2. Sugarbaker DJ. Macroscopic complete resection: the goal of primary surgery in multimodality therapy for pleural mesothelioma. J Thorac Oncol. 2006;1:175-6.

3. Batirel HF, Metintas M, Caglar HB, Ak G, Yumuk PF, Yildizeli B, et al. Adoption of pleurectomy and decortication for malignant mesothelioma leads to similar survival as extrapleural pneumonectomy. J Thorac Cardiovasc Surg. 2016;151: 478-84.

4. Kim DJ, Lee JG, Lee CY, Park IK, Chung KY. Long-term survival following pneumonectomy for non-small cell lung cancer: clinical implications for follow-up care. Chest. 2007;132:178-84. 